TITRE: LE MODÈLE DE RÉPONSE À L'INTERVENTION ET LA PRÉVENTION DES DIFFICULTÉS D'APPRENTISSAGE DE LA LECTURE AU PRÉSCOLAIRE ET AU PRIMAIRE

Auteur(s): Alain Desrochers, Université d’OTtaWA, Line LAPLANTE, UniVERSité du QuÉBec À MontréAL ET MONIQUe BROdEUR, UniVERSITÉ DU QuÉBEC À MONTRÉA

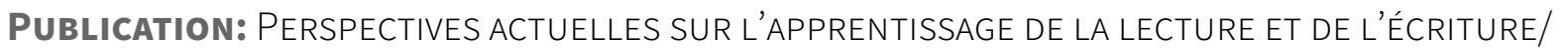
CONTRIBUTIONS ABOUT LEARNING TO READ AND WRITE - ACTES DU SYMPOSIUM INTERNATIONAL SUR LA LITÉRACIE À L'ÉCOLE/INTERNATIONAL SYMPOSIUM FOR EDUCATIONAL LITERACY (SILE/ISEL) 2015

PAGES: $290-314$

Directeurs: Marie-France Morin, Denis Alamargot et Carolina Gonçalves.

É́dITEUR: LES ÉDITIONS DE L'UNIVERSITÉ DE SHERBROOKE, 2016.

ISBN: 978-2-7622-0355-4

URI: HTTP://HDL.HANDLE.NET/11143/10274

DOI: HTTPS://DOI.ORG/10.17118/11143/10274 


\title{
Le modèle de réponse à l'intervention et la prévention des difficultés d'apprentissage de la lecture au préscolaire et au primaire
}

\author{
Alain Desrochers, Université d'Ottawa, \\ Line Laplante, Université du Québec à Montréal \\ Monique Brodeur, Université du Québec à Montréal
}

Résumé : La recherche scientifique en éducation montre qu'il est possible de réduire le nombre des élèves qui présentent des difficultés sévères d'apprentissage de la lecture en remplaçant l'approche « attendre l'échec avant d'agir » par une approche axée sur la prévention de l'échec. Le modèle de la réponse à l'intervention constitue une approche préventive dont l'efficacité a été attestée par des procédés scientifiques. Dans ce texte, nous décrivons d'abord les opérations proposées dans ce modèle pour prévenir les difficultés d'apprentissage et pour hausser l'engagement et la réussite des élèves. Ces opérations incluent la mise en œuvre de pratiques pédagogiques démontrées efficaces par la recherche, le dépistage régulier des élèves à risque ou en difficulté et l'intensification progressive des interventions pour répondre aux besoins de ces élèves, notamment par la mise en œuvre de pratiques orthopédagogiques. Ensuite, nous détaillons l'actualisation de ces opérations. Enfin, nous discutons les conditions essentielles à l'application réussie de ce modèle, selon trois phases : la planification, la réalisation, ainsi que l'évaluation d’impact et l'amélioration continue.

Mots clés: apprentissage de la lecture, maternelle, primaire, prévention des difficultés d'apprentissage, dépistage des élèves à risque ou en difficulté, intensification des interventions enseignantes et orthopédagogiques.

Abstract: Scientific research in education confirms that it is possible to reduce the number of children who struggle with learning to read by replacing the « wait-to-fail » approach by an approach focused on the prevention of failure. The Response-to-Intervention model offers a preventive approach to reading instruction that has been shown to be effective. This paper first describes the operations proposed to prevent learning difficulties and enhance learners' engagement and success. These operations include the implementation of evidence-based teaching practices, the regular screening of children at-risk or facing difficulties, and the progressive intensification of supplemental interventions to address their needs. Then, the detail of these operations is discussed. Finally, we summarize the conditions that are essential to a successful application of the model, namely, planning, implementation, impact assessment and continuous improvement.

Key words: reading learning process, kindergarten, primary school, learning difficulties prevention, detection of the students at risk and in difficulty, intensification of teaching interventions. 


\section{Introduction}

L'école, telle que nous la connaissons en Occident, s'est façonnée sous la pression de plusieurs facteurs, dont la Réforme protestante, la Contre-Réforme catholique, l'objectif de modeler les attitudes et les comportements des jeunes, la nécessité de former une main-d'œuvre mieux qualifiée pour soutenir le développement économique. Dès le $17^{e}$ siècle, l'enseignement collectif est instauré dans une salle de classe et assorti de recommandations pour gérer l'emploi du temps des élèves, l'espace qu'ils occupent dans l'école, leur posture corporelle, leurs déplacements et leurs conduites. En amont de la Révolution industrielle, un puissant incitatif à la scolarisation primaire obligatoire, un vaste réseau d'écoles et de collèges est constitué en Europe, et un programme de formation et de certification des enseignants est mis en œuvre. Cette mouvance est à l'origine des premières écoles normales, de l'évaluation et de l'approbation du matériel d'enseignement par un conseil d'éducation, de la programmation de l'enseignement et de la standardisation des manuels scolaires. Ce modèle de scolarisation remplace le tutorat individuel par un enseignement collectif pour la plupart des élèves (Gauthier, 2012; Maynes, 1985). Au moment où le roi Frédéric II de Prusse (1740 - 1786) rend l'instruction primaire obligatoire par des décrets $(1763,1765)$, et la reine Marie Thérèse d'Autriche (1740 - 1780) par un édit (1774), les écoles commencent à se constituer en un système éducatif intégré et un modèle d'éducation de masse est déjà largement balisé (Van Horn Melton, 1988). Au cours du $19^{e}$ siècle, ce mouvement vers l'instruction publique obligatoire et la démocratisation des savoirs de base se répand en Europe et conduit à un recul progressif de l'illettrisme (Hamerow, 1983, p. 169).

Dans ce modèle d'éducation de masse, les enseignants titulaires d'une classe sont désormais confrontés au défi de gérer l'hétérogénéité de leurs élèves sur le plan des aptitudes aux apprentissages scolaires et sur le plan de la motivation à effectuer ces apprentissages à l'école. Se pose alors le problème de conduire efficacement des apprentissages collectifs en faisant travailler tous les élèves en même temps et de soutenir les plus faibles. Comment, dans un tel modèle de scolarisation, pouvait-on éviter l'échec des plus faibles, sachant que le temps d'instruction est collectivement limité et que les élèves n'apprennent pas tous au même rythme? Les résultats issus des enquêtes menées depuis plus de 20 ans dans les pays de l'Organisation de coopération et de développement économiques (OCDE) donnent à penser que l'objectif de la littératie pour tous, malgré d'importantes innovations pédagogiques, n'est toujours pas atteint au terme de la scolarisation obligatoire. Par exemple, les résultats de l'enquête de 2012 sur la compétence à lire indiquent que 16,5\% des adultes canadiens de 16 ans ou plus se situent au niveau 1 ou sous ce niveau sur l'échelle (en 5 points) de littératie des populations, ce qui correspond à l'illettrisme fonctionnel; $32 \%$ des adultes canadiens se situent au niveau 2 et peinent à comprendre une phrase simple à l'écrit (Statistique Canada, 2013, p. 87). C'est dire que près de $50 \%$ des adultes canadiens démontrent un faible niveau de littératie après une dizaine d'années de scolarisation obligatoire. Le bilan est similaire dans tous les pays qui ont participé à l'enquête de 2012. Or, cette situation ne survient pas soudainement à l'âge de 16 ans. La recherche montre que, dès la $1^{\text {re }}$ année du primaire, l'apprentissage de la lecture et la persistance scolaire sont compromis chez les élèves qui ne font pas les progrès attendus (Janosz, Pascal, 
Belleau, Archambault, Parent et Pagani, 2013). Ces observations nous ramènent au défi de gérer les apprentissages dans les classes hétérogènes et de faire cheminer tous les élèves dans leur parcours scolaire (Cavallo et Chartier, 1997; Chartier, 2007). Dans la suite de notre exposé, nous donnons un aperçu des principes et des aménagements proposés dans le modèle de la réponse à l'intervention, un modèle axé sur la prévention des difficultés d'apprentissage ${ }^{1}$, et nous discutons quelques-unes des conditions qui contribuent à l'efficacité de sa mise en œuvre.

\section{Le modèle de la réponse à l'intervention : principes et application}

Le modèle de la réponse à l'intervention s'appuie sur un ensemble de principes qui nécessitent une opérationnalisation. La direction du National Center on Response to Intervention (NCRTI) résume ainsi notre compréhension actuelle de ce modèle :

[Le modèle de] la réponse à l'intervention réunit des procédés d'évaluation et d'intervention, dans un système axé sur la prévention et constitué de paliers multiples, pour maximiser la réussite des élèves et pour réduire les problèmes de comportement. Dans ce modèle, les écoles s'appuient sur des données pour identifier les élèves à risque de difficultés d'apprentissage, pour pister les progrès des élèves, pour mettre en œuvre des interventions démontrées efficaces par la recherche, pour adapter l'intensité et la nature de ces interventions à la réponse de l'élève à l'enseignement dispensé et pour identifier les élèves qui présentent un trouble d'apprentissage ou tout autre trouble (NCRTI, 2010, p. 2, notre traduction).

Cette description met en relief les composantes essentielles du modèle. D'abord, il s'agit d'un modèle systémique qui vise à faire progresser tous les élèves dans le parcours défini par le programme de formation. Pour atteindre cet objectif, on propose a) d'adopter des pratiques enseignantes dont l'efficacité réelle a été démontrée par la recherche, b) de mener un dépistage universel régulièrement pour identifier les élèves à risque de difficultés d'apprentissage ou ceux qui présentent déjà de telles difficultés, c) d'adapter les modalités d'intervention pédagogique à la réponse de chaque élève à l'enseignement dispensé, d) de mesurer les progrès des élèves pour vérifier l'impact de l'intensification des interventions pédagogiques et e) d'appuyer les décisions relatives aux élèves sur des données plutôt que sur des impressions (Brown-Chidsey et Steege, 2010; Broxterman et Whalen, 2013; Whitten, Esteves et Woodrow, 2012). Précisons que ces leviers éducatifs faisaient l'objet d'une attention bien avant la conception du modèle de la réponse à l'intervention². Sur le plan conceptuel, l'apport principal de ce modèle consiste à réunir, dans une approche cohérente et intégrative, des leviers éducatifs dont l'efficacité a été attestée par des procédés de démonstration issus de la recherche scientifique et de la pratique sur le terrain (Vaughn, Wanzek, Woodruff et Linan-Thompson,

1. Même si l'apprentissage de la lecture est en relation étroite avec celui de l'écriture, nous centrerons notre exposé exclusivement sur la lecture.

2. Ces leviers sont évoqués dans la politique de l'adaptation scolaire du ministère de l'Éducation du Québec sans qu'on fasse explicitement référence au modèle de la réponse à l'intervention (MÉQ, 1999). 
2007; Whitten et al., 2012). Sur le plan pratique, ce modèle s'inscrit dans une approche qui privilégie la collaboration entre tous les acteurs du milieu scolaire et l'amélioration continue des pratiques pédagogiques, d'évaluation et de gestion pour assurer la réussite scolaire des élèves (Broxterman et Whalen, 2013; Leclerc, 2012; Prud'Homme et Leclerc, 2014).

\section{Le concept de palier d'intervention}

La réponse à l'intervention renvoie à un régime pédagogique constitué de plusieurs paliers d'intervention gradués. La variante la plus courante comprend trois paliers (en anglais, three-tier model; pour une représentation schématique, voir la figure 1, adaptée de Whitten et al., 2012). Chaque palier d'intervention est caractérisé par la taille du groupe auquel il s'adresse (la classe entière, un petit groupe d'élèves) et des modalités d'intervention particulières. Les modalités d'intervention sont guidées par un protocole standardisé et dynamique. La standardisation vise à garantir que la mise en œuvre des interventions est conforme aux modalités qui ont été démontrées efficaces par la recherche. L'actualisation dynamique renvoie à l'ajustement des paramètres de l'intervention en fonction des progrès réalisés par les élèves.

Le palier I de la graduation désigne les pratiques éducatives universelles dispensées à l'ensemble des élèves de la classe. Ces pratiques englobent les prescriptions du programme de formation établi, des activités d'apprentissage et le matériel didactique afférent ainsi que le recours au dépistage universel des élèves à risque de difficultés d'apprentissage. Au palier I, on s'attend à ce que l'enseignement cible toutes les composantes essentielles de la compétence à lire et qu'il soit si efficace que la majorité des élèves ( $80 \%$ - $85 \%$ ) deviennent des lecteurs compétents sans intervention supplémentaire. Pour atteindre ce niveau d'efficacité, les enseignants doivent mettre en œuvre des pratiques éducatives (p.ex. choix d'un modèle d'enseignement et du matériel didactique) dont l'efficacité a été démontrée par des procédés scientifiques.

Le palier II s'adresse aux élèves qui accusent un retard dans leur apprentissage malgré un enseignement de haute qualité au palier I. Son objectif est d'assurer le développement des habiletés sous-jacentes à la compétence à lire de façon à ce que les élèves à risque ou en difficulté puissent éventuellement atteindre les cibles prévues dans le programme de formation. II s'agit donc d'un programme d'intervention supplémentaire et intensif offert aux élèves dont les progrès en lecture sont jugés insuffisants pour assurer leur réussite scolaire. Le palier III, pour sa part, constitue un programme d'intervention supplémentaire encore plus intensif et ciblé à l'intention des élèves pour qui le palier II n'a pas réussi à soutenir la progression des apprentissages attendus. Chaque palier s'inscrit donc sur un continuum d'intensification (palier I $\rightarrow$ palier $\| \rightarrow$ palier III) afin de répondre adéquatement aux besoins de tous les élèves et d'éviter qu'ils accumulent des retards insurmontables dans leurs apprentissages (Harn, Kame'ennui et Simmons, 2007; Vaughn, Denton et Fletcher, 2010). 
Il est à noter que l'enchaînement typique des trois paliers d'intervention n'est pas toujours optimal. Al Otaiba, Connor, Folsom, Wanzek, Greulich, Schatschneider et Wagner (2014) montrent que les élèves de $1^{\text {re }}$ année qui présentent des déficits importants sur le plan de certaines habiletés hautement associées à la réussite des premiers apprentissages en lecture font des progrès plus importants s'ils reçoivent dès le début de l'année scolaire, en supplément des interventions mises en œuvre au palier I, des interventions au palier II ou au palier III (voir aussi Vaughn et al., 2010). C'est pourquoi ces auteurs considèrent qu'il n'y a pas d'avantages à retarder l'intensification des interventions auprès des élèves déjà jugés hautement à risque.

Avant de détailler les modalités de chaque palier d'intervention, précisons que l'optimisation du modèle fait toujours l'objet d'une attention soutenue de la part des chercheurs en éducation. À ce stade, les recherches scientifiques sur l'optimisation des paliers I et II sont plus abondantes que sur celle du palier III (Al Otaiba et al., 2014; Gersten, Compton, Connor, Dimino, Santoro, Linan-Thompson et Tilly, 2009). Seules quelques études ont évalué l'effet du palier III à l'intérieur d'une même année scolaire, consécutivement aux paliers I et II (Al Otaiba et al., 2014; Fien, Smith, Smolkowski, Baker, Nelson, et Chaparro, 2015; Gilbert, Compton, Fuchs, Fuchs, Bouton, Barquero et Cho, 2013), et elles concernent typiquement les élèves de la maternelle à la $2^{\mathrm{e}}$ année du primaire (Compton et al., 2012; Toste, Compton, Fuchs, Fuchs, Gilbert, Cho, Barquero et Bouton, 2014). Les autres études ont surtout évalué l'effet du palier III après une année d'intervention aux paliers I et II (Denton et al., 2013). C'est dire que, dans l'état actuel des connaissances scientifiques, plusieurs questions restent encore ouvertes quant aux modalités optimales de chaque palier. En revanche, le modèle lui-même offre un cadre bien structuré pour guider autant la recherche scientifique que l'expérimentation sur le terrain.

\section{Un enseignement universel efficace de la lecture au palier I}

Le palier I est défini par le recours à des pratiques éducatives démontrées efficaces par la recherche à l'intention de tous les élèves de la classe. La préoccupation pour un enseignement collectif efficace de la lecture n'est pas récente. Au cours des siècles, plusieurs méthodes et dispositifs ont été proposés (Cavallo et Chartier, 1997) sans qu'on fasse appel à des procédés scientifiques pour en démontrer l'efficacité. À présent, une partie importante des recherches en éducation porte sur l'analyse empirique de l'efficacité des pratiques éducatives. Les pratiques dont l'efficacité a été démontrée sont retenues en priorité dans le modèle de la réponse à l'intervention.

Un consensus semble déjà largement établi sur la finalité de l'enseignement de la lecture : c'est-àdire conduire les apprenants à s'approprier toutes les habiletés nécessaires à la compréhension et à l'utilisation de la langue écrite selon les objectifs qu'ils poursuivent. Pour accéder à la signification d'un texte ou pour produire un texte, les apprenants doivent joindre deux grands ensembles d'habiletés, notamment celles qui président à la compréhension de la langue orale (p.ex. l'évocation du sens des mots et des phrases dans les communications orales) et celles qui concernent le traitement 
de la langue écrite (p.ex. l'identification visuelle des mots, le traitement des marques morphologiques de l'écrit). Paradoxalement, l'histoire récente de l'enseignement de la lecture est marquée par une opposition entre des modèles qui privilégient soit les habiletés propres au traitement du code orthographique, soit celles propres au traitement du sens véhiculé par l'écrit (Chall, 1983). Nous ne discuterons pas l'origine de cette opposition. Nous nous limiterons à rappeler que la compétence à lire une langue alphabétique comme le français résulte de la coordination d'habiletés propres à la langue écrite (p.ex. l'identification visuelle des mots et des marques morphologiques), d'habiletés partagées entre la langue orale et la langue écrite (p.ex. l'évocation du sens des mots, le traitement de l'organisation de la phrase) et d'habiletés cognitives générales (p.ex. le contrôle attentionnel, le stockage et la récupération des informations en mémoire, le raisonnement). Il faut donc se garder d'adopter une vue réductrice de cette compétence complexe.

Les experts qui se sont interrogés récemment sur les caractéristiques de l'enseignement efficace de la lecture ont appuyé leurs conclusions surtout sur des procédés de démonstration scientifiques et sur des résultats de recherche reproductibles (Brown-Chidsey et Steege, 2010). À titre d'illustration, relevons six des recommandations qui ont été rapportées dans divers ouvrages de synthèse (Denton, 2008; National Reading Panel, 2000; Pressley et Allington, 2015; Snow, Burns et Griffin, 1998; Spear-Swerling, 2015) : 1) assurer la préparation des enfants à l'apprentissage de la lecture; 2) centrer l'enseignement de la lecture sur les notions, les habiletés et les stratégies qui sont directement pertinentes au développement de cette compétence; 3) privilégier un modèle d'enseignement systématique et explicite pour assurer les apprentissages fondamentaux en lecture chez tous les élèves; 4) fournir aux élèves des occasions nombreuses et régulières de mettre en pratique les notions, les habiletés et les stratégies en lecture de textes signifiants avec le soutien de l'enseignant; 5) pister régulièrement les progrès des élèves et reprendre l'enseignement au besoin; et 6) utiliser les données issues du dépistage universel pour différencier l'enseignement et l'adapter aux besoins des élèves qui présentent des difficultés. Dans la suite de notre exposé, nous détaillons ces recommandations pour en extraire les éléments essentiels.

Préparation à l'apprentissage de la lecture. L'apprentissage de la lecture s'appuie sur des habiletés qui ont souvent atteint un niveau de développement relativement élevé au moment où les enfants entrent à la maternelle. Les activités langagières pratiquées dans l'environnement social des enfants auront pu les conduire à bien prononcer les mots de la langue orale et à hausser la richesse de leur vocabulaire, la complexité des phrases qu'ils peuvent interpréter ou produire ainsi que la variété de leurs stratégies communicatives. Les études sur les habiletés préalables à l'apprentissage de la lecture rapportent toutefois de grandes différences interindividuelles associées au statut socioéconomique des familles (Phillips et Lonigan, 2007). Pour réduire ces différences, les experts ont proposé de privilégier, dès la maternelle, les activités préparatoires à l'apprentissage formel de la lecture : le développement de l'expression orale, des concepts associés à l'écrit, de la prise de conscience des sons distinctifs de la parole et des connaissances alphabétiques (Pressley et Allington, 2015). 
Notions, habiletés et stratégies essentielles. Pour les langues alphabétiques, comme le français, les notions essentielles à l'apprentissage de la lecture renvoient aux bases de connaissances propres à la langue parlée et à la langue écrite telles que les phonèmes de la langue orale, les correspondances graphème-phonème, l'orthographe des mots, les procédés de construction des mots ou des phrases et le sens des mots isolés ou en contexte de phrase. Les habiletés désignent les savoir-faire propres à la lecture tels que l'identification visuelle des mots connus et l'évocation du sens de l'écrit. Les stratégies sont des plans d'action mis en œuvre délibérément par le lecteur pour surmonter des difficultés particulières telles que la lecture d'un mot inconnu ou l'interprétation d'une phrase difficile. Selon le National Reading Panel (2000), l'enseignement efficace de la lecture doit être centré sur le développement des habiletés qui lui sont essentielles : la différenciation et la manipulation des phonèmes, l'application des correspondances graphème-phonème pour identifier les mots écrits (décodage), l'accès à un vocabulaire étendu, la mise en œuvre de stratégies de compréhension de l'écrit et la fluidité (ou la fluence) des processus qui président à ces habiletés. Pour le français et les autres langues syntaxiquement plus complexes que l'anglais, il faut ajouter, à cette liste, la maîtrise de la grammaire, qui sous-tend l'organisation des phrases et le jeu des marques morphosyntaxiques.

Enseignement efficace. Les modèles d'enseignement sonttypiquementassortis de dispositifs et de recommandations pour appuyer les apprentissages scolaires. Ceux-ci peuvent différer considérablement quant au rôle dévolu à l'enseignantet à celui confiéauxélèves. Pourassurer des progrès soutenus danslesapprentissagesfondamentauxcheztouslesélèves, on recommandeauxenseignantsd'accorder environ90minutesparjouràl'enseignementdelalectureetd'adopterun modèled'enseignementaxésur une progression systématique des apprentissages et sur des instructions claires et explicites (Gauthier, Bissonnette et Richard, 2013; Hollingsworth et Ybarra, 2012). L'enseignement systématique se traduit d'abord par une mise en séquence ordonnée des objets d'apprentissage, qu'il s'agisse des correspondances graphème-phonème, des notions relatives aux procédés de construction des mots ou des phrases ou de la mise en relation des mots qui définissent un champ sémantique. Cette séquence constitue la base d'une progression des apprentissages qui permet aux élèves d'asseoir des notions ou des habiletés complexes sur la maîtrise de notions ou d'habiletés plus simples et, surtout, de favoriser l'intégration de toutes les habiletés qui sous-tendent la compétence en lecture. Les instructions dites explicites peuvent prendre plusieurs formes (p.ex. explications, directives, consignes, démonstrations), mais leur but est toujours le même : conduire tous les élèves à maîtriser une notion (p.ex. les correspondances graphème-phonème, la liaison, la ponctuation, l'accord), un savoir-faire (p.ex. la segmentation des graphèmes d'un mot, la conversion graphème-phonème, la lecture orale ou silencieuse d'un mot ou d'une phrase, l'inférence d'une information à la lecture d'un texte) ou l'application d'une stratégie. 
Pratique régulière et fréquente. La pratique assidue de la lecture constitue une condition essentielle à son apprentissage et à sa maîtrise. Aux élèves qui affrontent des difficultés, il est recommandé de leur fournir des occasions régulières et fréquentes de lire en pratique guidée et en pratique autonome (Denton et Hocker, 2006). La pratique guidée est particulièrement efficace lorsque la rétroaction fournie par l'enseignant souligne les actions que les élèves réussissent et lorsqu'elle signale les erreurs et permet d'éviter qu'une action incorrecte ne s'imprègne. Il est également recommandé d'offrir régulièrement aux élèves des périodes libres pendant lesquelles ils peuvent s'adonner à la pratique autonome de la lecture, alors que l'enseignant est présent pour offrir de l'aide. Le cumul des périodes de pratique constitue une condition importante pour consolider les progrès accomplis et pour assurer le développement de l'efficience en lecture.

Lecture de textes. Pour devenir des lecteurs compétents, il est essentiel que les élèves s'exercent, sous la supervision attentive de l'enseignant, à appliquer leurs habiletés et leurs stratégies à la lecture de textes, préférablement des textes décodables et signifiants. Les textes dits décodables sont ceux qui contiennent une proportion élevée de mots que les apprentis lecteurs peuvent identifier à partir des correspondances graphème-phonème qu'ils connaissent. Ces textes permettent aux apprentis lecteurs de lire une proportion élevée des mots qu'ils rencontrent et de tirer de ce succès une confiance accrue en leurs capacités, tout en leur offrant l'occasion de s'exercer à résoudre les problèmes associés aux mots difficiles à identifier. Les textes dits signifiants désignent ceux dont le contenu est à la portée des apprentis lecteurs. La principale caractéristique de ces textes est de soutenir la fonction première de la lecture, c'est-à-dire celle de lire pour s'informer, pour apprendre, ou simplement pour le plaisir. Le recours aux textes décodables et signifiants vise à conduire les élèves à réussir dans l'application de leurs habiletés et de leurs stratégies plutôt qu'à se décourager devant la complexité de la tâche de comprendre ce qu'ils lisent. Rappelons que les stratégies nécessaires à l'identification ou à l'interprétation des mots en contexte de phrases peuvent être enseignées explicitement et systématiquement (p.ex. Denton et Hocker, 2006; Klingner, Vaughn et Boardman, 2007).

La mise en œuvre de toutes ces recommandations ne suffit ordinairement pas à conduire tous les élèves à faire les gains attendus en lecture. Malgré un enseignement de qualité, une proportion des élèves n'arrivera pas à effectuer les mêmes apprentissages que leurs pairs dans la classe. Ces élèves sont parfois faciles à identifier, mais ce n'est pas toujours le cas. C'est pourquoi il est recommandé de faire appel à une procédure qui permet de suivre de près la progression de chaque élève en lecture.

\section{Le dépistage régulier des élèves à risque ou en difficulté au palier I}

Environ 80 \% des élèves réussissent à devenir des lecteurs compétents consécutivement à l'enseignement collectif de qualité offert au premier palier seulement (Spear-Swerling, 2015). Environ $15 \%$ ont besoin d'une intervention supplémentaire et ciblée (au palier II) et environ $5 \%$ présentent des difficultés si sévères qu'il est nécessaire de leur offrir un soutien rééducatif (au palier III)3. Pour assurer

3. Ces pourcentages sont des estimations théoriques. Dans les milieux défavorisés sur le plan socioéconomique, le pour- 
l'identification précoce des élèves à risque de difficultés d'apprentissage ou déjà aux prises avec ces difficultés, il est recommandé de mener un dépistage universel, à la maternelle et au primaire, au moins trois fois par année (p.ex. au début, au milieu et à la fin de l'année scolaire; Gilbert, Compton, Fuchs et Fuchs, 2012). Les habiletés ciblées par l'instrumentation servant au dépistage doivent être étroitement reliées au programme de formation puisque l'objectif de cette opération est d'identifier les élèves qui ne répondent pas adéquatement à l'enseignement dispensé au premier palier. C'est pourquoi les épreuves qui constituent les trousses de dépistage continu sont dites axées sur le curriculum (en anglais, curriculum-based; Hosp, Hosp et Howell, 2007; McMaster, Parker et Jung, 2012). Un des enjeux principaux d'une opération de dépistage consiste à classer les élèves avec justesse. Les vrais positifs désignent les élèves qui sont identifiés comme étant à risque et qui feront face à des difficultés si aucune intervention préventive n'est mise en œuvre. Les vrais négatifs sont ceux qui ne sont pas identifiés comme étant à risque et qui seront à l'abri de difficultés sévères ultérieurement. Deux autres catégories représentent des erreurs de classement. Les faux positifs, par exemple, sont les élèves qui sont identifiés comme étant à risque par l'instrumentation utilisée, mais qui ne présenteront aucune difficulté sévère ultérieurement. Les faux négatifs désignent les élèves qui ne sont pas identifiés comme étant à risque, mais qui le sont. Cette dernière catégorie est particulièrement préoccupante, car elle représente les élèves qu'on prive de service parce qu'on ne les juge pas à risque. C'est pourquoi il est fortement recommandé de faire appel à des outils d'évaluation qui répondent à des standards éducométriques élevés sur les plans de la fidélité (assurant ainsi la justesse et la stabilité de la mesure) et de la validité (ciblant ainsi le concept choisi; Fuchs et Fuchs, 2007).

Comment alors détermine-t-on qu'un élève est à risque de difficultés en lecture? Deux approches sont couramment utilisées dans le milieu scolaire en Amérique du Nord (Fuchs et Fuchs, 2007; Spear-Swerling, 2015). La première consiste à établir des standards de rendement attendus (appelés benchmarks en anglais) à différents moments dans l'année scolaire et à comparer le rendement de chaque élève à ces standards. Par exemple, on estime que les élèves qui peuvent lire oralement plus de 40 mots par minute dans un texte à la fin de la $1^{\text {re }}$ année de scolarisation présentent un risque réduit de manifester des difficultés en lecture orale ultérieurement (Good et Kaminski, 2002). Une autre approche consiste à se référer à un cadre normatif dont la fonction est de préciser le score à

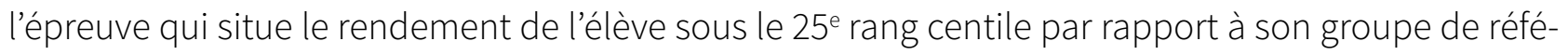
rence. Les données de dépistage, souvent recueillies par les enseignants, sont typiquement jointes à d'autres informations pour dresser un portrait de classe et pour déterminer les besoins des élèves jugés à risque. Il s'agit là d'une opération de synthèse instructive, car les élèves à risque n’ont pas tous les mêmes besoins. L'appréciation de ces besoins est déterminante dans la planification de l'intervention supplémentaire la plus appropriée pour un élève.

centage des élèves qui nécessitent une intervention au palier II ou III est souvent plus élevé que celui que nous rapportons ici. 


\section{L'intensification des interventions aux paliers II et III}

Le modèle à trois paliers, comme nous le soulignions plus haut, constitue la variante la plus courante du modèle de la réponse à l'intervention et la mieux documentée (Vaughn et al., 2010). Le palier I (intervention universelle) constitue la pierre angulaire du modèle. Son but est d'assurer la réussite en lecture de la majorité des élèves en leur offrant un enseignement de haute qualité. Le but du palier II et du palier III est d'assurer le développement de certaines habiletés sous-jacentes à la compétence à lire de façon à ce que les élèves à risque, ou qui présentent des difficultés, puissent éventuellement réussir leurs apprentissages. Pour différencier les modalités propres aux paliers II et III, examinons deux classes de caractéristiques qui leur sont propres.

Caractéristiques organisationnelles. Nous traitons d'abord de quatre caractéristiques organisationnelles dans l'opérationnalisation des paliers II et III. La première concerne la personne qui prend l'intervention en charge à chaque palier d'intensification. Dans la majorité des études réalisées aux États-Unis, ce sont les enseignants titulaires de la classe ou les enseignants spécialistes de contenu (p.ex. en lecture) qui interviennent au palier II (Denton, 2012). Au palier III, la tâche revient ordinairement aux enseignants spécialisés ou aux spécialistes de matière ou de contenu (Harn et al., 2007). Dans les études réalisées au Québec, les interventions au palier II et au palier III sont typiquement confiées à l'orthopédagogue (Brodeur, Laplante, Desrochers et Dion, 2015; Desrochers, Laplante, Brodeur, et DesGagné, 2015; Laplante et al., 2014). Ce choix est justifié par la nécessité d'un niveau minimal de spécialisation pour assurer la mise en œuvre des interventions adaptées aux élèves à risque ou déjà en difficulté.

La seconde caractéristique organisationnelle se rapporte au lieu où sont dispensées les interventions (Denton, 2012). Ainsi, au palier II, la classe constitue le lieu où se déroulent les interventions lorsque c'est l'enseignant titulaire qui en a la charge. Si ces interventions sont assurées par l'enseignant spécialiste de matière (la lecture) ou l'orthopédagogue, les interventions sont ordinairement mises en œuvre dans un local à l'extérieur de la classe. Les interventions au palier III se déroulent typiquement dans un local à l'extérieur de la classe.

La troisième caractéristique organisationnelle se rapporte au ratio intervenant/apprenant dans l'intensification des interventions. Dans les études américaines sur l'effet du palier II, ce ratio varie généralement entre 4 et 8 élèves (Al Otaiba et al., 2014; Brown-Chidsey et Steege, 2010). Au palier III, le ratio se situe typiquement entre 1 à 3 élèves par intervenant (Chard et Harn, 2008; Harn et al., 2007). Qu'il s'agisse du palier II ou du palier III, il est recommandé de grouper les élèves sur la base de besoins similaires tout en prenant en compte la classe d'appartenance pour faciliter la gestion des périodes d'enseignement et du déplacement des élèves dans l'école. Ainsi, les activités d'apprentissage ciblées peuvent profiter à tous les élèves du groupe. 
La quatrième et dernière caractéristique organisationnelle concerne la structure temporelle des interventions mises en œuvre. Encore peu d'études ont porté sur le dosage de l'intervention : le nombre de semaines d'intervention, le nombre de séances par semaine, la durée de chaque séance (Compton et al., 2012). Les paramètres décrits ci-après sont donc présentés à titre indicatif. Les interventions offertes au palier II sont habituellement dispensées de 3 à 5 fois par semaine, à raison d'environ 30 à 40 minutes par séance (Compton et al., 2012; Vaughn et al., 2007). La période d'intervention s'étend de 8 à 15 semaines, voire à 20 semaines ou plus dans certains cas (Fuchs, Fuchs et Compton, 2004; Wanzek et Vaughn, 2007). Selon Denton et ses collaborateurs (2011), si on estime que le but poursuivi au palier II est « [...] d'accélérer le progrès des élèves qui requièrent un soutien supplémentaire à l'enseignement de qualité dispensé en classe afin de leur permettre d'atteindre un niveau de lecture conforme à leur niveau scolaire [...] (p. 226) », il est préférable d'augmenter le nombre d'heures d'intervention (au minimum 32 heures). Par contre, si cet objectif est d'identifier les élèves qui ont besoin d'un soutien encore plus intensif, au palier III, la mise en œuvre de ce second palier devrait être considérée comme étant temporaire et de plus courte durée (au maximum 32 heures). À cet égard, certains auteurs suggèrent que le palier II soit implanté par cycles de 8 semaines, au terme desquels le progrès de chaque élève est évalué afin de déterminer s'il a besoin ou non d'un autre cycle d'interventions au palier II, ou si une intensification plus forte est requise (au palier III).

L'intensification offerte au palier III se traduit naturellement par une augmentation de la fréquence des séances d'intervention - de 4 à 5 fois par semaine, à raison de 45 à 60 minutes par séance - et par une augmentation de la durée totale de l'intervention (Vaughn et al., 2007). À ce palier, le nombre de semaines d'intervention n'est pas fixe; il varie selon le degré de sévérité des difficultés rencontrées. Notons que très peu d'études ont évalué l'impact des interventions au palier III dans la foulée de celles mises en œuvre aux paliers I et II, et ces études sont caractérisées par des interventions réparties sur un nombre relativement restreint de semaines (8 semaines dans Sanchez et O'Connor, 2015; 8 semaines dans Laplante et al., 2016), une durée qui pourrait être insuffisante selon la sévérité des difficultés des élèves. À titre d'exemple, dans l'étude réalisée par Denton et ses collaborateurs (2013), des élèves de $2^{\mathrm{e}}$ année, dont le niveau de réponse à l'intervention aux paliers I et II avait été jugé insuffisant lorsqu'ils étaient en 1re année, ont reçu des interventions intensives au palier III à raison de 5 séances par semaine, de 45 minutes chaque séance, sur une période de 24 à 26 semaines, pour une moyenne de 75,6 heures d'intervention. Au terme de cette intervention, les élèves ont fait des progrès sur plusieurs plans : décodage, identification de mots (justesse et fluidité), compréhension de phrases et de passages de texte. Toutefois, en dépit des progrès observés, plusieurs d'entre eux peinaient encore à apprendre à lire. Ces non-répondants présentaient toujours des faiblesses importantes sur le plan de la conscience phonologique, de la dénomination des lettres et de la compréhension orale. Ces résultats mettent en relief l'importance d'évaluer adéquatement la complexité des difficultés auxquelles les élèves sont confrontées, car la planification des interventions en dépend. Malgré une planification rigoureuse, les progrès des élèves en difficulté très sévère ne sont pas toujours garantis. C'est pourquoi il est impératif de poursuivre la recherche scientifique sur les caractéristiques des élèves qui affrontent de grandes difficultés et sur les interventions éducatives. 
Caractéristiques pédagogiques et didactiques. La première caractéristique dont nous traitons concerne la priorisation des cibles essentielles. Les cibles d'un enseignement efficace de la lecture aux paliers II et III sont de la même nature que celles traitées au palier I; c'est-à-dire qu'on recommande de centrer l'intervention sur les notions, sur les habiletés et sur les stratégies qui agissent directement sur la compétence à lire et de privilégier un modèle d'enseignement systématique et explicite pour soutenir ces apprentissages (Denton, Fletcher, Taylor, Barth et Vaughn, 2014; Weiser et Mathes, 2011). Le nombre de cibles retenues aux paliers II ou III est toutefois moindre qu'au palier I. Les cibles choisies correspondent ordinairement aux habiletés les plus déficitaires chez l'élève. L'intensification du soutien à l'apprentissage se traduit donc par des choix sur les plans pédagogique et didactique.

La seconde caractéristique se rapporte aux modalités de gestion des apprentissages. Consécutivement à une analyse des études qui ont évalué l'effet d'interventions offertes au palier II sur l'apprentissage de la lecture, Fien et ses collaborateurs (2015) observent que la mise en œuvre de l'enseignement explicite est bonifiée par une augmentation : 1) du modelage par l'intervenant des habiletés et des stratégies ciblées, 2) des occasions offertes à l'élève de mettre en pratique ces habiletés et ces stratégies au cours d'une séance (Vaughn et al., 2007), 3) de la rétroaction immédiate et des explications fournies par l'intervenant (Gunn, Smolkowski, Biglan, Black et Blair, 2005) et 4) du rythme avec lequel les activités sont animées par l'intervenant afin de favoriser le développement de plusieurs habiletés au cours d'une séance (Vaughn et al., 2010). Relativement au palier II, l'enseignement dispensé au palier III est encore plus explicite, centré sur la remédiation d'un nombre plus restreint d'habiletés, lesquelles ont été sélectionnées sur la base des données recueillies, notamment lors du pistage des progrès réalisés au palier II. La rétroaction y est également plus systématique et adaptée aux difficultés rencontrées par l'élève. Le palier III est donc davantage orienté vers un soutien individualisé, en réponse aux besoins spécifiques de chaque élève (Sanchez et O’Connor, 2015).

La troisième caractéristique concerne les habiletés spécifiques visées au palier II. Une riche documentation scientifique appuie la conclusion que l'enseignement explicite est particulièrement efficace lorsqu'il cible la conscience phonémique, les correspondances graphème-phonème et la lecture de textes avec rétroaction de l'enseignant. On observe alors, chez les élèves, une amélioration importante de leur capacité à identifier les mots écrits avec justesse et fluidité (Blachman et al., 2004; Mathes et al., 2005). La lecture répétée de textes, avec modelage et rétroaction, est aussi un moyen efficace d'améliorer la fluidité en lecture de texte, en particulier chez les élèves du primaire (Lee et Yoon, 2016). Enfin, l'enseignement explicite de stratégies de compréhension qui conduisent les lecteurs en difficulté à accorder une attention particulière au sens véhiculé par le texte, telles que l'identification de l'idée principale et la synthèse, conduit à une amélioration significative de leur performance en lecture (Berkeley, Scruggs, et Mastropieri, 2010; Solis, Ciullo, Vaughn, Pyle, Hassaram et Leroux, 2012). 
Au troisième palier, les habiletés ciblées par l'intervention varient en fonction du portrait des élèves. À titre d'exemple, dans l'étude de Sanchez et O'Connor (2015), l'intervention mise en œuvre au palier III avait pour cible l'analyse et l'identification de mots écrits et la fluidité, étant donné que les élèves présentaient un déficit important sur le plan de ces habiletés. Dans le cas de l'étude de Denton et ses collaborateurs (2013), des activités visant l'identification de mots écrits, la fluidité en lecture orale, les stratégies de compréhension faisaient partie de l'éventail des possibilités; l'intervenant ajustait quotidiennement ses choix en fonction des besoins et des progrès des élèves. Dans le cadre d'une étude réalisée au Québec en français (Laplante et al., 2016), les interventions offertes aux élèves de $1^{\text {re }}$ année présentant des difficultés importantes d'apprentissage de la lecture visaient à améliorer la justesse et la fluidité de l'identification des mots écrits puisque ces habiletés continuaient d'être largement déficitaires en dépit des interventions mises en œuvre au premier et au second palier depuis le début de l'année scolaire.

Les modalités d'implantation des paliers II et III mettent en relief la nécessité de divers aménagements sur le plan organisationnel et sur le plan de la qualification du personnel scolaire. Or, toutes les conditions facilitantes ne sont pas nécessairement présentes au moment de la mise en œuvre du modèle de la réponse à l'intervention dans un environnement scolaire particulier.

\section{Les conditions nécessaires à une mise en œuvre efficace du modèle}

L'impact réel de la mise en œuvre du modèle de la réponse à l'intervention dépend de plusieurs facteurs tels le niveau de préparation des intervenants, la rigueur de l'implantation du modèle, l'efficacité des interventions et l'adéquation des procédés d'évaluation (Denton, 2012; Spear-Swerling, 2015). Dans cette section, nous faisons un retour sur les conditions nécessaires à une mise en œuvre efficace du modèle de la réponse à l'intervention et nous les déclinons selon les trois étapes inhérentes à l'autorégulation : la planification, la réalisation, ainsi que l'évaluation d'impact et l'amélioration continue (Brodeur, Dion, Mercier, Laplante et Bournot-Trites, 2009; Zimmerman, 2000).

\section{La planification}

La planification d'une mise en œuvre efficace du modèle de réponse à l'intervention nécessite la mobilisation de l'ensemble des acteurs scolaires (la direction de l'établissement, les enseignants, les spécialistes) et un plan d'action détaillé (une scénarisation des opérations, une répartition explicite des responsabilités). La mobilisation présuppose une prise de conscience collective du fait que les élèves arrivent à l'école avec une préparation aux apprentissages scolaires qui est fort variable, voire lacunaire dans certains cas, et que plusieurs d'entre eux ne réussissent pas à apprendre à lire adéquatement au terme de leurs études primaires et secondaires. De plus, les élèves qui présentent des difficultés sévères en lecture à la fin de la $1^{\text {re }}$ année du primaire sont plus à risque que les autres de continuer à éprouver des difficultés au secondaire, de décrocher de l'école et de faire face à un 
parcours de vie semé d'embûches, que ce soit sur le plan de l'employabilité, de la santé et de la participation à la société (Institut de la statistique du Québec , 2006).

Le recours à des pratiques dont l'efficacité a été démontrée par des procédés scientifiques est essentiel pour répondre aux besoins des élèves, et le modèle de la réponse à l'intervention réunit plusieurs de ces pratiques. Les acteurs scolaires doivent aussi croire en leur propre capacité d'apprendre à mettre en œuvre ces pratiques et de mieux soutenir les élèves dans leurs apprentissages. Les acteurs conscients de ces enjeux sont plus aptes à se mobiliser et à fournir les efforts nécessaires pour actualiser un plan d'action (Brodeur, Perreault, Ouellet et Desrochers, 2011). Noltemeyer, Boone et Sansosti (2014) ont identifié six variables déterminantes dans l'élaboration et l'actualisation d'un plan d'action. Nous les évoquons brièvement.

Leadership. Les efforts requis par la mise en œuvre du modèle de la réponse à l'intervention proviennent d'abord du leadership et du soutien des cadres scolaires (Fullan, 2014). Ce leadership dans la mobilisation des équipes-écoles est particulièrement efficace lorsqu'il s'exerce dans le respect de l'autonomie professionnelle et dans un climat de collaboration (Broxterman et Whalen, 2013).

Développement professionnel. L'adoption du modèle de la réponse à l'intervention conduit typiquement à des changements importants dans les pratiques relatives à l'enseignement, à l'évaluation des élèves et à la gestion scolaire. Or, il se trouve que les intervenants peuvent être conduits à assumer des responsabilités pour lesquelles ils ont peu de préparation. C'est pourquoi le développement professionnel doit faire l'objet d'une attention particulière et faire partie intégrante du plan d'action. Par exemple, la formation initiale des enseignants peut être lacunaire relativement aux concepts afférents à la langue écrite ou aux pratiques démontrées efficaces par la recherche (Barrio et Combes, 2014). Cette observation est importante, car le premier niveau de prévention des difficultés d'apprentissage dans ce modèle se trouve au palier I. Alors que le développement professionnel au palier I gagne à être offert à l'ensemble des acteurs, celui relatif aux paliers II et III est typiquement réservé à des enseignants ayant reçu une formation spécialisée. Au Québec, il s'agit des orthopédagogues dont la formation les rend plus aptes à dispenser des interventions adaptées aux besoins particuliers des élèves à risque ou en difficulté.

Efficacité des pratiques enseignantes. L'identification et l'adoption de pratiques pédagogiques et orthopédagogiques démontrées efficaces, idéalement selon des paramètres qui ont été validés, constituent des opérations déterminantes dans le déploiement des leviers éducatifs. L'identification de ces pratiques représente un défi, car il existe peu d'instances pouvant les recenser, les évaluer et les recommander. Quant à leur utilisation, il s'agit également d'un défi, car on observe un écart significatif, bien que variable, entre les pratiques démontrées efficaces et celles mises en œuvre dans les écoles (Kretlow et Helf, 2013). 
Adéquation des pratiques d'évaluation. L'identification des élèves à risque de difficultés en lecture et des élèves déjà confrontés à ces difficultés renvoie à trois procédés d'évaluation distincts : le dépistage, le pistage des progrès et l'évaluation orthopédagogique (Whitten et al., 2012). L'exploitation de ces procédés nécessite une instrumentation et des savoir-faire particuliers. Les ressources relatives à l'instrumentation sont essentielles à la mise en œuvre du modèle de la réponse à l'intervention. Bien qu'elles soient particulièrement abondantes en anglais, il en existe en français, notamment pour le dépistage continu (Desrochers et Bonneau, 2014) et pour l'évaluation détaillée des difficultés en lecture (pour un compte rendu, voir Desrochers, DesGagné et Kirby, 2011). De plus, pour assurer l'adéquation des pratiques évaluatives, il est impératif de former les intervenants à l'utilisation de cette instrumentation, à la gestion des données et à la prise de décision guidée par des données (Prud'homme et Leclerc, 2014).

Prise de décision guidée par des données. Une des dispositions les plus importantes du modèle de la réponse à l'intervention consiste à utiliser les données recueillies auprès des élèves pour guider les décisions à leur sujet. Cette pratique vise à réduire le poids des impressions subjectives ou erronées dans les décisions prises par l'équipe-école. L'une de ces décisions est de déterminer qui sont les élèves à risque d'échec en lecture. L'estimation de ce risque est une opération délicate, car elle est sujette à deux types d'erreurs : la détection d'élèves qui ne sont pas réellement à risque (qu'on appelle faux positifs) et la non-détection des élèves qui sont effectivement à risque (qu'on appelle faux négatifs). Le premier cas d'erreur conduit à offrir à un élève des services (une intervention au palier II) dont il n'a pas besoin et le deuxième à priver un élève de services dont il a réellement besoin. Une autre décision consiste à déterminer si les élèves à risque ou en difficulté font des progrès réels consécutivement à une intervention au palier II ou III, ou s'il est indiqué de mettre fin à cette intervention. Il est recommandé de s'appuyer sur des données dans ces décisions (Prud’homme et Leclerc, 2014).

Fidélité de la mise en œuvre du modèle. Comme nous le soulignions plus haut, l'impact réel du modèle de la réponse à l'intervention dépend étroitement de la qualité du plan d'action, mais tout autant de la rigueur de sa mise en œuvre. Un bon plan d'action n'a d'impact que s'il est bien exécuté. Plusieurs moyens ont été proposés en éducation pour assurer la fidélité de la mise en œuvre d'un modèle d'intervention : la formation et l'accompagnement des intervenants, la consignation des actions réalisées (dans un journal de bord) et le bilan annuel des réalisations, de leur impact réel et des opérations à améliorer (Kretlow, Cooke et Wood, 2012; Meyers et Brandt, 2014).

\section{La réalisation}

Tout au long de la mise en œuvre du modèle de la réponse à l'intervention, les six variables déterminantes doivent faire l'objet d'une veille afin de s'assurer de la qualité de l'implantation du modèle. La direction de l'école doit poursuivre l'exercice de son leadership, de même que son soutien au projet et aux intervenants. Les enseignants et les orthopédagogues doivent pouvoir compter sur 
un accompagnement lors de la mise en œuvre des paliers d'intervention éducative, du dépistage continu, du pistage des progrès des élèves, de la gestion des données d'évaluation et de la prise de décision afférente.

\section{L'évaluation d'impact et l'amélioration progressive}

L'exécution d'un plan d'action en éducation est rarement parfaite à cause de la complexité des opérations requises et de la complexité de l'environnement dans lequel ces opérations sont réalisées. Les écarts entre le plan d'action et l'exécution de ce plan peuvent réduire considérablement l'impact réel d'un projet éducatif. C'est pourquoi les acteurs scolaires doivent faire une évaluation juste, sans complaisance, de leurs réalisations et de leur impact réel sur les élèves (les apprentissages, la motivation) et l'ensemble de leur milieu de travail (le sentiment d'efficacité des intervenants). Cette évaluation permet d'identifier les facteurs qui ont été favorables et les obstacles, de même que les émotions ressenties, de façon à apporter les améliorations nécessaires au plan d'action ou à son exécution lors du prochain cycle. Elle favorise également l'adoption et le maintien des nouvelles pratiques des enseignants et des orthopédagogues. Enfin, il faut prévoir que tous les acteurs n'auront pas eu la même facilité à mettre en œuvre les pratiques attendues, pour des raisons personnelles, contextuelles ou organisationnelles, et que des modalités de soutien seront nécessaires (Brodeur et al., 2008; Klingner, 2004). Dans tous les cas, la persévérance des acteurs est une condition essentielle à l'amélioration progressive de la mise en œuvre du modèle et à l'atteinte des objectifs fixés.

\section{Conclusion}

La prévention des difficultés d'apprentissage de la lecture représente un enjeu majeur pour l'école, qui a la responsabilité d'assurer l'égalité des chances entre les élèves, de leur permettre de développer pleinement leur potentiel et de les préparer à la vie d'adulte. Le modèle de la réponse à l'intervention représente l'un des cadres de référence les mieux documentés et prometteurs pour réunir et coordonner un vaste ensemble de leviers éducatifs dans le milieu scolaire. Notre objectif, dans cet exposé, était d'expliquer les principales opérations proposées dans ce modèle et de rassembler les conditions essentielles à l'efficacité de son implantation.

Si plusieurs ouvrages ont été publiés en anglais sur le modèle de la réponse à l'intervention, la documentation en français est encore rare. Notre exposé constitue une synthèse ponctuelle sur le sujet. Les résultats des travaux, lors desquels des enseignants et des orthopédagogues ont mis en place dans leur milieu de travail le modèle de la réponse à l'intervention, accompagnés par des chercheurs et des conseillers pédagogiques, démontrent la faisabilité et l'efficacité de sa mise en œuvre, notamment en milieu très défavorisé. II reste toutefois un travail important à accomplir sur le plan de la recherche, du développement (matériel pour l'intervention aux trois paliers, outils d'évaluation valides et fiables) et du transfert des connaissances vers les milieux de pratique. Pour accélérer la réalisation de ce travail, nous préconisons la coordination et la collaboration entre les acteurs de la 
recherche et ceux du terrain, dans le cadre de projets nationaux et internationaux. Cette approche favorise le développement efficace et le partage des ressources éducatives et de l'expertise, contribuant ainsi à un enrichissement collectif au profit des élèves, des acteurs scolaires, des systèmes éducatifs et de la société. 


\section{Références}

Al Otaiba, S., Connor, C. M., Folsom, J.S., Wanzek, J., Greulich, L., Schatschneider, C. et Wagner, R.K. (2014). To wait in Tier 1 or intervene immediately: A randomized experiment examining first-grade response to intervention in reading. Exceptional Children, 81(1), 11-27.

Barrio, B. L. et Combes, B. H. (2014). General education pre-service teachers' levels of concern on response to intervention (RTI) implementation. Teacher Education and Special Education, 1-17.

Berkeley, S., Scruggs, T. E. et Mastropieri, M. A. (2010). Reading comprehension instruction for students with learning disabilities, 1995-2006 : A meta-analysis. Remedial and Special Education, 31(6), 423-436.

Blachman, B. A., Schatschneider, C., Fletcher, J. M., Francis, D. J., Clonan, S., Shaywitz, B. et Shaywitz, S. (2004). Effects of intensive reading remediation for second and third graders. Journal of Educational Psychology, 96, 444-461.

Brodeur, M., Dion, E., Mercier, J., Laplante, L. et Bournot-Trites, M. (2009). Le rôle et la formation des enseignants et des orthopédagogues pour l'apprentissage de la lecture. Stratégie nationale d'alphabétisation précoce. London, ON : Réseau canadien de recherche sur le langage et l'alphabétisation.

Brodeur, M., Laplante, L., Desrochers, A. et Dion, É. (2015, août). Effet de l'implantation du modèle de réponse à l'intervention sur le développement de la conscience phonologique et des connaissances alphabétiques à la maternelle. Communication au Symposium International sur la Littératie à l'École (SILE), Orford, Canada.

Brodeur, M., Laplante, L., Dion, É., Godard, L., Gosselin, C., Mercier, J., Vanier, N., Campeau, M. Ė., Lapierre, M., Fournier, K. et Potvin, M. C. (2008). La forêt de l'alphabet, programme de prévention des difficultés d'apprentissage en lecture pour la maternelle. Programme de Niveau I dans le cadre du Modèle d'intervention multiniveaux ou de réponse à l'intervention. Guide pédagogique. Traduction et adaptation de l'Optimize Intervention Program. Montréal : Centre de Psycho-Éducation du Québec (1 édition : 2006).

Brodeur, M., Perreault, M., Ouellet, C. et Desrochers, A. (2011). L'analphabétisme crée l'obligation d'agir. Dans L'État du Québec 2011, Le Québec est-il (toujours) une société égalitaire ? Montréal : Boréal, p. 373-380.

Brown-Chidsey, R. et Steege, M. W. (2010). Response to intervention: Principles and strategies for effective practice. New York : Guilford Press.

Broxterman, K. et Whalen, A. J. (2013). RTI team building: Effective collaboration and data-based decision making. New York: Guilford Press.

Cavallo, G. et Chartier, R. (1997). (Dir.), Histoire de la lecture dans le monde occidental. Paris : Le Seuil.

Chall, J. S. (1983). Learning to read: The great debate. New York : McGraw-Hill. 
Chard, D. et Harn, B. (2008). Project CIRCUITS: Center for Improving Reading Competence Using Intensive Treatments Schoolwide. Dans C. Greenwood, T. Kratochwill et M. Clements (dir.) Schoolwide Prevention Models: Lessons Learned in Elementary Schools (p. 70-83). New York: Guilford Publications.

Chartier, A.-M. (2007). L'école et la lecture obligatoire : histoire et paradoxes des pratiques d'enseignement de la lecture. Paris : Retz.

Compton, D. L., Gilbert, J. K., Jenkins, J. R., Fuchs, D., Fuchs, L. S., Cho, E., Barquero, L. A. et Bouton, B. (2012). Accelerating chronically unresponsive children to Tier 3 instruction: What level of data is necessary to ensure selection accuracy? Journal of Learning Disabilities, 45(3), 204-216.

Denton, C. A. (2008). Classroom reading instruction that supports struggling readers: Key components for effective teaching. Site internet : http://www.rtinetwork.org/essential/tieredinstruction/ tier1/effectiveteaching.

Denton, C. A. (2012). Response to intervention for reading difficulties in the primary grades: Some answers and lingering questions. Journal of Learning Disabilities, 45(3), 232-243.

Denton, C. A., Cirino, P. T., Barth, A. E., Romain, M., Vaughn, S., Wexler, J., ...Fletcher, J. M. (2011). An experimental study of scheduling and duration of "Tier 2" first-grade reading intervention. Journal of Research on Educational Effectiveness, 4(3), 208-230.

Denton, C. A., Fletcher, J. M., Taylor, W. P., Barth, A. E. et Vaughn, S. (2014). An experimental evaluation of guided reading and explicit interventions for primary-grade students at-risk for reading difficulties. Journal of Research on Educational Effectiveness, 7, 268-293.

Denton, C. A. et Hocker, J. L. (2006). Responsive reading instruction: Flexible intervention for struggling readers in the early grades. Longmount, $\mathrm{CO}$ : Sopris West.

Denton, C. A., Tolar, T. D., Fletcher, J. M., Barth, A. E., Vaughn, S. et Francis, D. J. (2013). Effects of Tier 3 intervention for students with persistent reading difficulties and characteristics of inadequate responders. Journal of Educational Psychology, 105(3), 633-648.

Desrochers, A. et Bonneau, M. (2014). Épreuves de fluidité en lecture : guide d'utilisation. Victoriaville: Commission scolaire des Bois-Francs.

Desrochers, A., DesGagné, L. et Kirby, J. R. (2011). L'évaluation de la lecture orale. Dans M. J. Berger et A. Desrochers (dir.), L'évaluation de la littératie (p. 177-214). Ottawa: Presses de l'Université d'Ottawa.

Desrochers, A., Laplante, L., Brodeur, M. et DesGagné, L. (2015, août). L'impact d'un modèle d'enseignement systématique à deux niveaux d'intensification sur l'apprentissage de la lecture orale et de l'orthographe en 1re année du primaire. Symposium International sur la Littératie à l'École (SILÉ), Orford, Canada.

Fien, H., M. Smith, J. L., Smolkowski, K., Baker, S. K., Nelson, N. J. et Chaparro, E. (2015). An examination of the efficacy of a multitiered intervention on early reading outcomes for first- grade students at risk for reading difficulties. Journal of Learning Disabilities, 48(6), 602-621. 
Fuchs, L. S. et Fuchs, D. (2007). The role of assessment in the Three-Tier approach to reading instruction. Dans D. Haager, J. Klingner et S. Vaughn (dir.), Evidence-based reading practices for response to intervention (p. 29-42). Baltimore, MD : Paul Brookes Publishing.

Fuchs, D., Fuchs, L. S. et Compton, D. L. (2004). Identifying reading disabilities by responsiveness-to-instruction: specifying measures and criteria. Learning Disbility Quarterly, 27(4), 216-227.

Fullan, M. (2014). Le leadership moteur. Comprendre les rouages du changement en éducation. La Fondation Lucie et André Chagnon. Corwin Press Inc.

Gauthier, C. (2012). Le XVII siècle et la naissance de la pédagogie. Dans C. Gauthier et M. Tardif (dir.), La pédagogie : théories et pratiques de l'Antiquité à nos jours (p. 59-75). Montréal : Gaëtan Morin Éditeur (3e édition).

Gauthier, C., Bissonnette, S. et Richard, M. (2013). Enseignement explicite et réussite des élèves : la gestion des apprentissages. Montréal : Pearson.

Gersten, R., Compton, D., Connor, C. M., Dimino, J., Santoro, L., Linan-Thompson, S. et Tilly, W. D. (2009). Assisting students struggling with reading: Response to Intervention and multi-tier intervention for reading in the primary grades. A practice guide. (NCEE \#2009-4045). Washington, DC: National Center for Education Evaluation and Regional Assistance, Institute of Education Sciences, U.S. Department of Education. Retrieved from http://ies.ed.gov/ncee/wwc/publications/practiceguides/

Gilbert, J. K., Compton, D. L., Fuchs, D. et Fuchs, L. S. (2012). Early screening for risk of reading disabilities: Recommendations for a four-step screening system. Assessment for effective intervention, 38(1), 6-14.

Gilbert, J. K., Compton, D. L., Fuchs, D., Fuchs, L. S., Bouton, B., Barquero, L. A. et Cho, E. (2013). Efficacy of a first-grade responsiveness-to-intervention prevention model for struggling readers. Reading Research Quarterly, 48(2), 135-154.

Good, R. H. et Kaminski, R. A. (2002). Dynamic Indicators of Basic Early Literacy Skills (6e édition). Eugene, OR: Institute for the Development of Educational Achievement.

Gunn, B., Smolkowski, K., Biglan, A., Black, C. et Blair, J. (2005). Fostering the development of reading skill through supplemental instruction: Results for Hispanic and non-Hispanic students. Journal of Special Education, 39(2), 66-85.

Hamerow, T. S. (1983). The birth of a new Europe: State and society in the nineteenth century. Chapel Hill, NC: The University of North Carolina Press.

Harn, B. A., Kame'ennui, E. J. et Simmons, D. C. (2007). The nature and role of the third tier in a prevention model for Kindergarten students. Dans D. Haager, J. Klingner et S. Vaughn (dir.), Evidence-Based Reading Practices for Response to Intervention (p. 161-184). Baltimore, MD : Brookes.

Hollingsworth, J. et Ybarra, S. (2012). L'enseignement explicite: une pratique efficace. Montréal : Chenelière Éducation. 
Hosp, M. K., Hosp, J. L. et Howell, K. W. (2007). The ABCs of CBM: A practical guide to curriculum-based measurement. New York: Guilford.

Institut de la statistique du Québec (2006). Développer nos compétences en littératie : un défi porteur d'avenir. Rapport québécois de l'Enquête internationale sur l'alphabétisation et les compétences des adultes (EIACA), 2003.

Janosz, M., Pascal, S., Belleau, L., Archambault, I., Parent, S. et Pagani, L. (2013). Les élèves du primaire à risque de décrocher au secondaire: caractéristiques à 12 ans et prédicteurs à 7 ans. Québec : Institut de la statistique du Québec.

Klingner, J. K. (2004). The science of professional development. Journal of Learning Disabilities, 37(3), 248-255.

Klingner, J. K., Vaughn, S. et Boardman, A. (2007). Teaching reading comprehension to students with learning difficulties. New York: Guilford.

Kretlow, A. G., Cooke, N. L. et Wood, C. L. (2012). Using inservice and coaching to improve the fidelity of evidence-based strategies. Remedial and Special Education, 33(6), 348-361.

Kretlow, A. G. et Helf, S. S. (2013). Teacher implementation of evidence-based practices in Tier 1 : A national survey. Teacher Education and Special Education, 36(3), 167-185.

Laplante, L., Desrochers, A., Brodeur, M., Chapleau, N., Fejzo, A., Godard, L., Mercier, J., Bédard, M. et Laguë, D. (2014, Mars). Impact du modèle RAl/multiniveaux sur la réussite des premiers apprentissages en littératie d'élèves de maternelle. Congrès de l'Association québécoise des troubles d'apprentissage (AQETA), Montréal, Québec.

Laplante, L., Mercier, J., Brodeur, M., Laguë, D. et Bédard, M. (2016). The impact of a secondary intervention on phonemic awareness, alphabetic knowledge and word identification of at-risk French-speaking children in Kindergarten. Communication au $23^{e}$ congrès de la Society for the Scientific Study of Reading. Porto, Portugal.

Leclerc, M. (2012). Communauté d'apprentissage professionnelle: guide à l'intention des leaders scolaires. Québec: Presses de l'Université du Québec.

Lee, J. L. et Yoon, S. Y. (2016). The effects of repeated reading on reading fluency for students with reading disabilities: A meta-analysis. Journal of Learning Disabilities, sous presse.

Mathes, P. G., Denton, C. A., Fletcher, J. M., Anthony, J. L., Francis, D. J. et Schatschneider, C. (2005). The effects of theoretically different intervention and student characteristics on the skills of struggling readers. Reading Research Quarterly, 40(2), 148-182.

Maynes, M. J. (1985). Schooling in Western Europe : A social history. New York: State University of New York.

McMaster, K. L., Parker, D. et Jung, P.-G. (2012). Using curriculum-based measurement for beginning writers within a response to intervention framework. Reading Psychology, 33(1-2), 190-216. 
Meyers, C. et Brandt, W. C. (2014). Implementation fidelity in education research: Designer and evaluator considerations. New York: Routledge.

Ministère de l'Éducation du Québec. (1999). Un école adaptée à tous ses élèves: politique de l'adaptation scolaire. Québec : Gouvernement du Québec.

National Center on Response to Intervention. (2010). Essential components of RTI - A closer look at response to intervention. Site Internet: http://www.rti4success.org/sites/default/files/rtiessentialcomponents_042710.pdf.

National Reading Panel. (2000). Teaching children to read: An evidence-based assessment of the scientific research literature on reading and its implications for reading instruction. Washington, DC: National Institute of Child Health and Human Development.

Noltemeyer, A. L., Boone, W. J. et Sansosti, F. J. (2014). Assessing school-level RTI implementation for reading: Development and piloting of the RTIS-R. Assessment for Effective Intervention, 40(1), 40-52.

Phillips, B. M. et Lonigan, C. J. (2007). Social correlates of emergent literacy. Dans M. J. Snowling et C. Hulme (dir.), The science of reading: A handbook (p. 173-187). Oxford, UK: Blackwell.

Pressley, M. et Allington, R. L. (2015). Reading instruction that works: The case for balanced teaching. New York: Guilford Press.

Prud'Homme, R. et Leclerc, M. (2014). Données d'observation et gestion de l'apprentissage : guide à l'intention des communautés d'apprentissage professionnelles. Québec : Presses de l'Université du Québec.

Sanchez, V. M. et O'Connor, R. E. (2015). Building Tier 3 intervention for long-term slow growers in Grades 3-4: A pilot study. Learning Disabilities Research \& Practice, 30(4), 171-181.

Snow, C. E., Burns, M. S. et Griffin, P. (1998). Preventing reading difficulties in young children. Washington, DC: National Academy Press.

Solis, M., Ciullo, S., Vaughn, S., Pyle, N., Hassaram, B. et Leroux, A. (2012). Reading comprehension interventions for middle school students with learning disabilities: A synthesis of 30 years of research. Journal of Learning Disabilities, 45(4), 327-340.

Spear-Swerling, L. (2015). The power of RTI and reading profiles : A blueprint for solving reading problems. Baltimore: Paul Brookes Publishing.

Statistique Canada. (2013). Les compétences au Canada: Premiers résultats du Programme pour l'évaluation internationale des compétences des adultes (PEICA). Ottawa, Canada: Ministère de l'Industrie.

Toste, J. R., Compton, D. L., Fuchs, D., Fuchs, L. S., Gilbert, J. K., Cho, E., Barquero, L. A. et Bouton, B. D. (2014). Understanding unresponsiveness to Tier 2 reading intervention: Exploring the classification and profiles of adequate and inadequate responders in first grade. Learning Disability Quarterly, 37(4), 192-203. 
Van Horn Melton, J. (1988). Absolutism and the eighteenth-century origins of compulsory schooling in Prussia and Austria. New York : Cambridge University Press.

Vaughn, S., Denton, C. A. et Fletcher, J. M. (2010). Why intensive interventions are necessary for students with severe reading difficulties. Psychology in the Schools, 47(5), 432-444.

Vaughn, S., Wanzek, J., Woodruff, A. L. et Linan-Thompson, S. (2007). A three-tier model for preventing reading difficulties and early identification of students with reading disabilities. Dans D. Haager, J. Klingner et S. Vaughn (dir.), Evidence-based reading practices for response to intervention (p. 1128). Baltimore: Brookes.

Wanzek, J. et Vaughn, S. (2007). Research-based implications from extensive early reading interventions. School Psychology Review, 36(4), 541-561.

Weiser, B. et Mathes, P. (2011). Using encoding instruction to improve reading and spelling performances of elementary students at risk of literacy difficulties : A best-evidence synthesis. Review of Educational Research, 81(2), 170-200.

Whitten, E., Esteves, K. J. et Woodrow, A. (2012). La réponse à l'intervention : un modèle efficace de différenciation. Montréal : Chenelière Éducation.

Zimmerman, B. J. (2000). Attaining self-regulation: a social cognitive perspective. Dans M. Boekaerts, P. R. Pintrich et M. Zeidner (dir.), Handbook of self-regulation (p. 13-39). San Diego : Academic Press. 


\section{Annexe 1}

Figure 1. Représentation schématique des principales caractéristiques distinctives des trois paliers du modèle de la réponse à l'intervention.

Palier III: Intervention intensive, supplémentaire et ciblée, en plus petits groupes ou individuelle, centrée sur des besoins spécifiques, avec pistage plus fréquent des progrès

Palier II: Intervention intensive et supplémentaire, en petits groupes, avec pistage fréquent des progrès

Palier I: Enseignement de qualité en classe, avec dépistage universel au moins trois fois par année

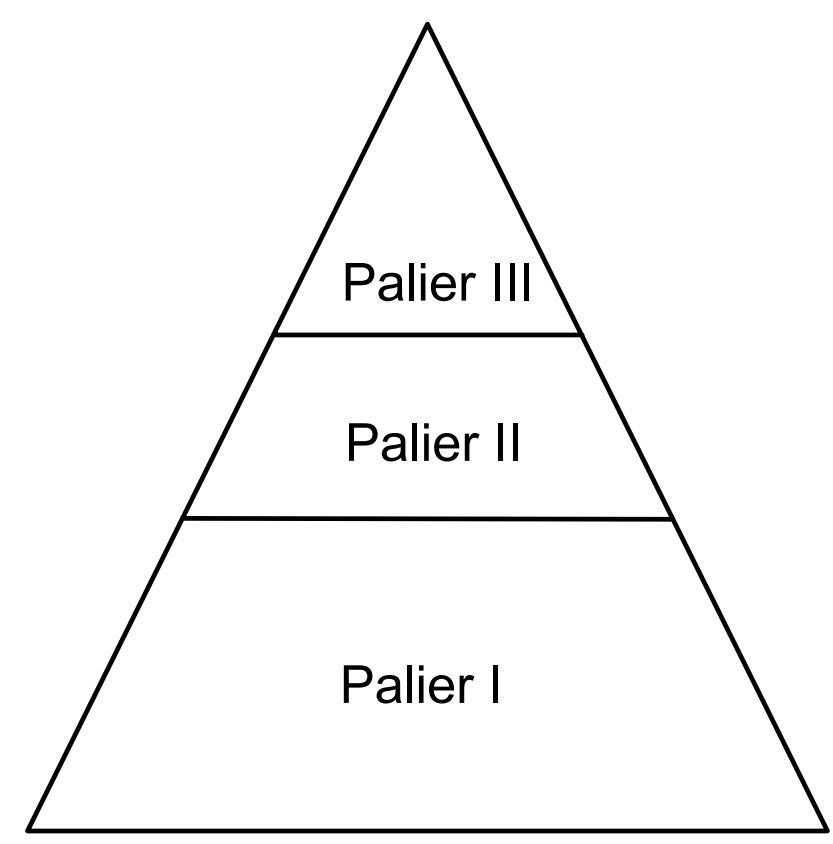

